\title{
The Down-Regulation of MicroRNA-497 Contributes to Cell Growth and Cisplatin Resistance Through PI3K/Akt Pathway in Osteosarcoma
}

\author{
Xue-jun Shao Mei-hua Miao Jun Xue Jian Xue Xue-qiang Ji Hong Zhu \\ Department of Clinical Laboratory Diagnosis, Soochow University Affiliated Children's Hospital of \\ Soochow University, Suzhou, China
}

\author{
Key Words \\ Osteosarcoma • microRNA-497 • Cisplatin • VEGFA
}

\begin{abstract}
Background: Down-expression of microRNA-497 (miR-497) was often found in malignancies. The purposes of this study were to determine the expression of miR-497 in human osteosarcoma and to establish the association between miR-497 expression with cell survival and the sensitivity to cisplatin in human osteosarcoma cells. Methods: The effects of ectopic miR-497 expression on the cell survival and cisplatin sensitivity in osteosarcoma cells were measured by the Cell Counting Kit-8 (CCK-8) assay. Quantitative real-time PCR (qRT-PCR) was utilized to determine the expression of miR-497. The effects of ectopic miR-497 expression on the expression of VEGFA, Akt and p-Akt were determined by western blot. Results: Realtime quantitative PCR analysis revealed that miR-497 was significantly down-regulated in osteosarcoma tissues and in the osteosarcoma cell line SAOS-2 compared with adjacent nontumorous osteosarcoma tissues and normal human osteoblasts. Up-regulation of miR497 inhibited cell survival and enhanced the sensitivity to cisplatin in osteosarcoma cells. In addition, knockdown of miR-497 induced osteosarcoma cells growth and cisplatin resistance. Luciferase reporter assay and western blot confirmed that VEGFA was a direct target of miR497. PI3K inhibitor LY294002 abrogated miR-497 inhibitors induced cisplatin resistance. Conclusion: Taken together, our results suggest that miR-497 modulates the sensitivity to cisplatin at least in part through PI3K/Akt pathway in osteosarcoma cells.
\end{abstract}

\section{Introduction}

Copyright (c) 2015 S. Karger AG, Basel

Osteosarcoma is the most common malignant bone tumor, comprising $2.4 \%$ of all malignancies in pediatric patients [1]. Patients are usually treated with aggressive chemotherapy before and after surgery. Improvement of new surgical techniques and chemo- and/or radiotherapy regimens resulted in a 5 year survival rate of about $60 \%$ [2].

X.-j. Shao and M.-h. Miao contributed equally to this article and should be considered co-first authors.

Hong Zhu

KARGER 125
Department of Clinical Laboratory Diagnosis, Children's Hospital of Soochow University, No 303, Jinde Road, Suzhou 215003 (China)

E-Mail biomed2003@163.com 
However, patients that do not respond to chemotherapeutic regimens have a poor prognosis. Moreover, the frequent acquisition of drug-resistant phenotypes and the occurrence of second malignancies associated with chemotherapy remain significant obstacles to achieving favorable outcomes. Thus, the analysis of the underlying mechanisms of chemoresistance in osteosarcoma cancer cells to chemotherapy is critical for the development of novel treatment strategies for this disease.

Cisplatin (CDDP) is still one of the most effective and widely used chemotherapeutic agents against osteosarcoma due to its therapeutic advantages, such as effectiveness, simple administration and mild side effects. However, CDDP resistance often occurs in clinical practice [3]. Recent studies have shown some microRNAs were dysregulated in several types of cancer and contributes to tumor resistance to chemotherapy. MiRNAs, which are approximately 21-25 nucleotides in length, are shown to act as post-transcriptional regulators of gene expression by binding to complementary sites in the 3'untranslated regions (3'UTRs) of the mRNAs [4]. Some miRNAs were found to be down- or up-regulated in cancer cells characteristic of a CDDP resistant phenotype, such as breast cancer cells [5], tongue squamous cell carcinoma [6] and germ cell tumors [7]. MiR-141 could counteract the apoptosis induced by CDDP in esophageal squamous cell carcinoma by targeting YAP1 [8]. On the contrary, up-regulation of miR-451 increases the sensitivity of non-small cell lung cancer cell line A549 to cisplatin [9], and miR-148a could modulate the sensitivity of esophageal cancer cell lines to cisplatin [10]. These studies together highlight the need to study miRNAs which play important roles in CDDP chemoresistance in various cancers.

The miR-497 and miR-195 genes are located on the short arm of chromosome 17, more precisely at 17p13.1 [11]. Flavin et al. found that miR-195/miR-497 were downregulated in primary peritoneal carcinoma [12]. MiR-195 was found downregulated in chronic lymphocytic leukemia, bladder, gastric, prostate carcinoma, and hepatocellular, while miR497 was found downregulated in human male breast carcinoma and prostate carcinoma [13]. In addition, Zhu et al. found that miR-497 was downregulated in human drug-resistant gastric and lung cancer cell lines, compared with their each parental cell lines [14]. Our previous work has shown that miR-497 is under-expressed in primary osteosarcoma tumors. However, the molecular details of miR-497 in osteosarcoma development and response of osteosarcoma cells to chemotherapy remain unclear. In this report, we shown that miR-497 targets VEGFA and is frequently downregulated in osteosarcoma. In addition, we demonstrated that downregulation of miR-497 was associated with elevated activation of PI3K/Akt signalling and cisplatin resistance in osteosarcoma cells.

\section{Materials and Methods}

\section{Patients and tissue samples}

A total of 14 primary osteosarcoma and corresponding noncancerous tissue samples from the same specimens were collected from the Department of Pathology, Soochow University Affiliated Children's Hospital between 2010 and 2013. Primary osteosarcoma and corresponding normal bone tissues were immediately frozen in liquid nitrogen and stored at $-70{ }^{\circ} \mathrm{C}$ until use. No patients had received chemotherapy, radiotherapy or blood transfusion before surgery. The clinicopathologic variables such as age, gender and the histologic type were retrospectively reviewed on the basis of the medical records. The samples were used with the written informed consent from patient and the approval of the ethic committee of Soochow University Affiliated Children's Hospital.

\section{Cell culture}

Human osteosarcoma cell line SAOS-2 cells were purchased from ATCC (American Type Culture Collection, MD, USA). The cells were cultured in Dulbecco's modified Eagle's Medium (Gibco RL, Grand Island, NY, USA) supplemented with $100 \mathrm{U} / \mathrm{ml}$ penicillin, $100 \mathrm{mg} / \mathrm{ml}$ streptomycin and $10 \%$ fetal bovine serum and were cultured at $37{ }^{\circ} \mathrm{C}$ in a humidified $5 \% \mathrm{CO}_{2}$ atmosphere. Normal human osteoblasts were isolated and cultured as previously described [15]. 


\section{Quantitative real-time RT-PCR}

Total RNA was extracted from cells or tissues using Trizol according to the manufacturer's protocol. Total RNA was reverse transcripted from 50ng total RNA with MMLV high performance reverse transcriptase using following primers: RT-miR-497: 5'-GTC GTA TCC AGT GCA GGG TCC GAG GTA TTC GCA CTG GAT ACG ACA CAA AC-3'; RT-U6:5'-CGCT TCACG AAT TTG CGT GTC AT-3'. miR-497 forward, 5'-GTG CAG GGT CCG AGG T-3', miR-497 reverse, 5'-TAG CCT GCA GCA CAC TGT GGT-3'; U6 forward,5'-GCT TCG GCA GCA CAT ATA CTA AAA T-3', U6 reverse, 5'-CGC TTC ACG AAT TTG CGT GTC AT-3'. Real-time PCR was performed using the standard SYBR Green Assay protocol by the 7500 Real-Time PCR System (Applied Biosystem). The $25 \mu \mathrm{L}$ PCR reaction included $2 \mu \mathrm{L}$ reverse transcription product, 1× PCR Master Mix (Takara, Dalian, China), $1 \mu \mathrm{mol} / \mathrm{L}$ forward primer and reverse primer. The PCR conditions were as follows: $95{ }^{\circ} \mathrm{C}$ for $3 \mathrm{~min}$, 35 cycles of $95{ }^{\circ} \mathrm{C}$ for 15 seconds, $60{ }^{\circ} \mathrm{C}$ for 30 seconds, $72{ }^{\circ} \mathrm{C}$ for 30 seconds. The comparative Ct method for relative quantification of gene expression was used to determine miRNA expression levels. Each sample was analyzed in triplicate, and the levels of U6 RNA were used to normalize the miRNA levels. VEGFA mRNA levels were examined as previously described [16].

\section{Oligonucleotides transfections}

SAOS- 2 cells were plated in 6-well plates $\left(6 \times 10^{5}\right.$ cells/well), $100 \mathrm{nM}$ of the miR-497 mimic, 100 $\mathrm{nM}$ of the miR-497 inhibitor or $100 \mathrm{nM}$ miRNA negative control was transfected into SAOS-2 cells using lipofectamine 2000 (Invitrogen, Long Island, NY, USA) according to the manufacturer's protocol, respectively. The miR-497 mimic, miR-497 inhibitor, and miRNA negative control were chemically synthesized by Shanghai GenePharma Company (Shanghai, China). The sequences of the miR-497 mimic, miR-497 inhibitor, and miRNA control were described previously [14].

Cell viability assay

SAOS-2 cells in logarithmic growth seeded at a density of $4 \times 10^{3}$ well into 96-well plates and were transfected with miR-497 mimic, miR-497 inhibitor or siVEGFA with their corresponding controls. After incubating the cells for the specified time, a cell proliferation assay was performed using Cell Counting Kit-8 (CCK-8) (Beyotime, Haimen, China) according to the manufacturer's instructions. The solution absorbance was measured spectrophotometrically at $450 \mathrm{~nm}$ with iMark $^{\mathrm{TM}}$ Microplate Absorbance Reader (Bio-Rad, Hercules, CA, USA). The experiments were performed in triplicate.

Colony formation assay

$2 \mathrm{ml}$ cell suspensions (1,000 cells) were seeded in six-well plates, into three repeat wells. The cells were then incubated for 14 days and fixed with $4 \%$ paraformaldehyde (Beyotime, Haimen, China) and stained with crystal violet (Sigma-Aldrich). The colony formations ( $\geq 50$ cells) were counted using an Olympus microscope IX73 (Olympus, Tokoyo, Japan).

Apoptosis Assay

The ratio of the total apoptotic cells was detected by the Annexin V-FITC apoptosis detection kit (Beyotime, Haimen, China). Briefly, after 72 hours treatment, the SAOS- 2 cells were collected and washed twice with ice-cold PBS buffer, re-suspended in $150 \mu \mathrm{L}$ of binding buffer, incubated with $10 \mu \mathrm{L}$ of Annexin $\mathrm{V}$ conjugated to FITC and $10 \mu \mathrm{L}$ PI for $20 \mathrm{~min}$ at room temperature, and analyzed by flow cytometry. Hoechst33258 was also used for the evaluation of the apoptosis. The cells were exposed to $5 \mu \mathrm{g} / \mathrm{ml}$ Hoechst 33258 for $15 \mathrm{~min}$ at room temperature under dark conditions. Subsequently, the apoptosis was detected under an Olympus fluorescence microscope IX73. Apoptotic cells were characterized by morphological changes, such as cell shrinkage, nuclear condensation, and fragmentation.

\section{Luciferase activity assay}

A fragment containing the 3'-UTR of human VEGFA (isoform a, NM_001025366.2), was amplified from human genomic DNA using the following primer pair: 5'-ATC TCA GCA TGC CTG GTC AGT TAC CTA CTA ATA GCG GGC CTG-3' and 5'-GCC CTG AGT GCT GAG CGA TCA AGT GTC ATT TGA CGT ATC GCT-3'. pGL3VEGFA-wild reporter was created by ligation of PCR products of 3'UTR of VEGF-A was cut with XbaI and cloned into the corresponding sites of the pGL3 vector. The QuikChange Site-Directed Mutagenesis Kit (Stratagene) was used to introduce the mutation in the putative miR-497 recognition element according 
to the manufacturer's recommendations. Cells were seeded in 24 -well plates at $5 \times 10^{4}$ cells per well the day before transfection. Luciferase reporter (500 ng), 50 pmol (miRNA-497 mimics or NC) and 40 ng of pRLTK Renilla Luciferase Reporter Vector (Promega) were added in each well. Cells were collected $48 \mathrm{~h}$ after transfection and luciferase activity was measured using Promega Dual-Luciferase ${ }^{\circledR}$ Reporter Assay system.

Western blot analysis

Proteins were separated by SDS-PAGE, transferred to polyvinylidene difluoride (PVDF) membranes (Millipore, Bedford, MA, USA). Blots were blocked with TBST containing 5\% non-fat dry milk for $1 \mathrm{~h}$ and then probed with antibodies against VEGFA(1: 1000 dilution; Abcam, Cambridge, MA, USA), AKT (1: 1000 dilution; Santa Cruz Biotechnology, CA, USA), phospho-AKT (Ser473; 1: 400 dilution; Santa Cruz Biotechnology, CA, USA), mouse anti-GAPDH, (1: 5000 dilution; Santa Cruz Biotechnology, CA, USA). After washing, the blots were incubated with horseradish peroxidase-conjugated secondary antibodies (Beyotime, Haimen, China) and visualized by super ECL detection reagent (Applygen, Beijing, China).

SiRNA transfection

The VEGFA siRNA (siVEGFA, sense strand 5'-CCG AAA CCA UGA ACU UUC UTT-3' and antisense strand: 5'-AGA AAG UUC AUG GUU UCG GTT-3') and scramble siRNA (sense strand $5^{\prime}$-UUC UCC GAA CGU GUC ACG UTT-3' and antisense strand: 5'-ACG UGA CAC GUU CGG AGA ATT-3') were synthesized by Shanghai GenePharma Co. (Shanghai, China). SAOS-2 cells were plated into 6-well plates and allowed to grow to 70 $\%$ confluent. Cells were transiently transfected with the siRNA using Lipofectamine ${ }^{\mathrm{TM}} 2000$ (Invitrogen, Carlsbad, CA) in OPTI-MEM medium (Gibco) according to the manufacturer's recommendations for $12 \mathrm{~h}$, and then incubated and used for further experiments.

Tumor xenograft model

In vivo experiments were performed in strict accordance with the guidelines of the National Laboratory Animal Center. SAOS-2 cells were pre-treated with the miR-449a mimics (50 nM) or NC. $1 \times 10^{7}$ transfected SAOS-2 cells were injected subcutaneously in the flanks of SCID mice as described previously [17]. The length and width of the tumors were measured every 5 days using a digital caliper and tumor volume was calculated as follows: large diameter $\times$ (small diameter $)^{2} \times 0.52$. After 35 days, tumor samples were carefully removed.

\section{Statistical Analysis}

Data are presented as mean \pm SD. All statistical analyses were performed using SPSS13.0 software. The differences between groups were compared using Student's t-test. Statistical difference was accepted at $\mathrm{P}<0.05$.

\section{Results}

MiR-497 is down-regulated in osteosarcoma tissue samples and osteosarcoma cell lines

The diagnosis of osteosarcoma was made by H\&E-stained samples from the patients (original magnification, $\times 100$ ) (Fig. 1A). Expression of miR-497 in 14 osteosarcoma tissue samples and matched non-tumor normal tissue samples were detected by qRT-PCR. MiR-497 was significantly down-regulated in osteosarcoma tissue samples compared with adjacent normal tissues (Fig. 1B). Similarly, expression of miR-497 in the osteosarcoma cell line SAOS2 was significantly decreased compared with that in normal human osteoblasts (Fig. 1C).

Overexpression of miR-497 inhibits, while knockdown of it promotes SAOS-2 cell growth

The proliferation rates of SAOS- 2 cells with enhanced or silenced expression of miR-497 were determined via CCK- 8 assay. After 72 hours of treatment, CCK- 8 assay was performed. SAOS- 2 cells with overexpression of miR-497 by transfected with miR-497 mimic significantly inhibited proliferation as compared with blank cells or cells transfected with the negative control, respectively (Fig. 2A and 2B). In contrast, miR-497 antisense oligonucleotide (ASO) transfected cells proliferated at a significantly higher rate as compared with blank cells or cells transfected with the negative control, respectively (Fig. 2C and 2D). Colony formation 
Fig. 1. MiR-497 is down-regulated in osteosarcoma tissue samples and osteosarcoma cell lines. A: The diagnosis of osteosarcoma was made by H\&Estained samples from the patients (original magnification, $\times 100$ ). B: MiR-497 expression in osteosarcoma tissue (T) with paired adjacent normal tissues (ANT) from 14 patients. C: Real-time PCR analysis of miR-497 expression in osteosarcoma cell line SAOS- 2 and normal human osteoblasts. ${ }^{*} P<0.05$.

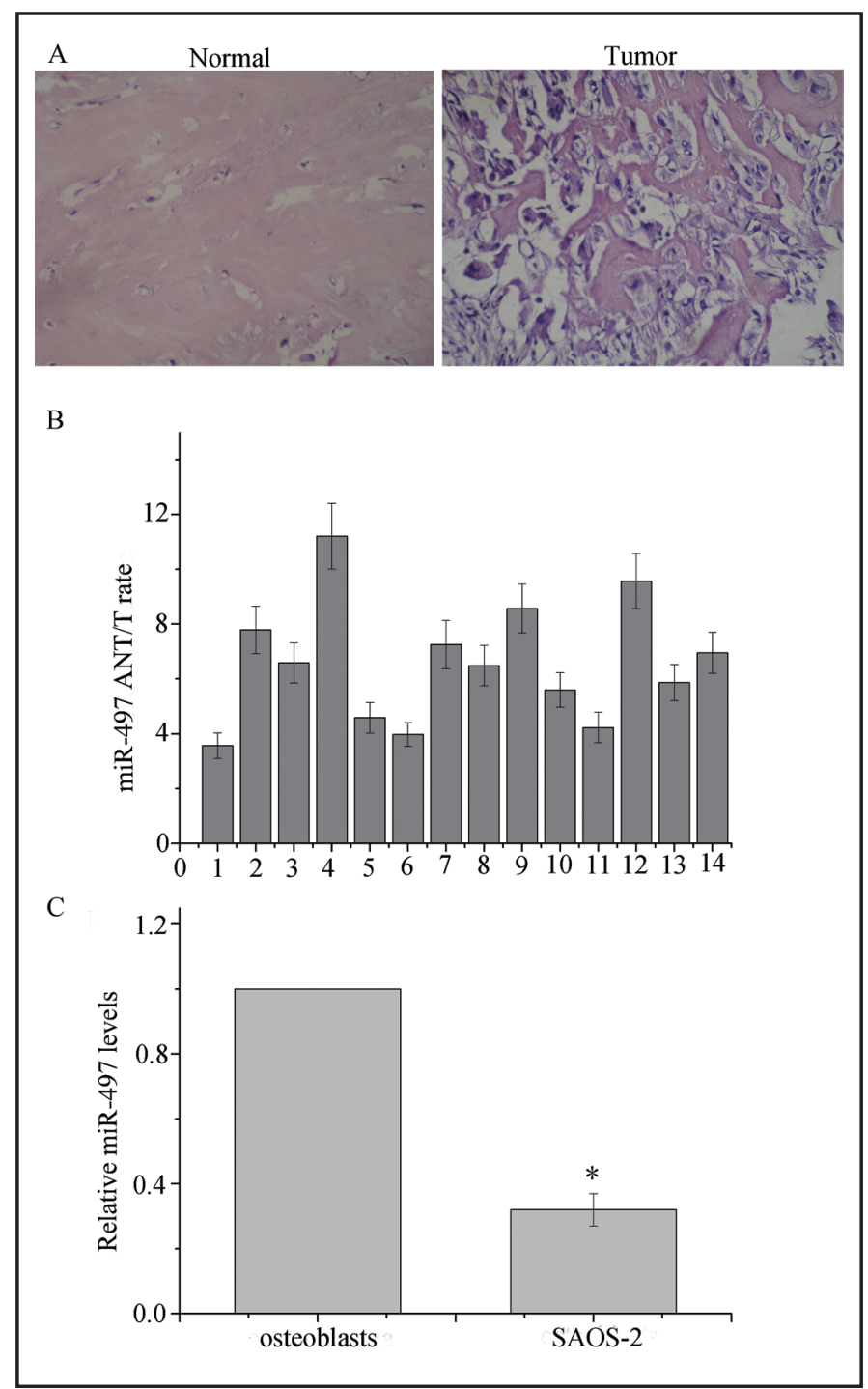

assay confirmed the results of CCK- 8 assay. As shown in Fig. 2E and 2F, overexpression of miR-497 inhibits colony formation ability of SAOS-2 cell. The results demonstrated that miR497 inhibited the proliferation of SAOS-2 cell.

Involvement of miR-497 in cisplatin resistance in SAOS-2 cells

Next, we examined whether miR-497 was capable of enhacing cisplatin-induced cytotoxicity. SAOS- 2 cells were transfected with miR-497 mimics or miR-497 inhibitors. The cells were treated with $2 \mu \mathrm{M}$ cisplatin for $48 \mathrm{~h}$. CCK-8 assay showed that compared with NC group, overexpression of miR-497 markedly increased cisplatin-induced cell cytotoxicity in SAOS-2 cells (Fig. 3A). Whereas the SAOS-2 cells transfected with the miR-497 inhibitors exhibited a significantly increasing resistance to CDDP compared with the controls (Fig. 3B). We quantified cisplatin-induced apoptosis of miR-497 mimics, miR-497 ASO and negative control transfected Saos-2 cells. As shown in Fig. 3C and 3D, miR-497 mimics transfected Saos- 2 cells treated with $2 \mu \mathrm{M}$ cisplatin had higher rates of apoptosis than the negative control transfected cells. But Saos-2 cells transfected with miR-497 ASO had lower rates of apoptosis than the negative control transfected cells. Similarly, the miR-497 mimics transfected group showed increased numbers of Hoechst 33258 positively stained cells $48 \mathrm{~h}$ after treatment, indicating an enhanced apoptotic activity. The results indicated that miR-497 was involved in cisplatin resistance in SAOS-2 cells.

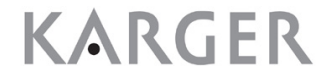


A

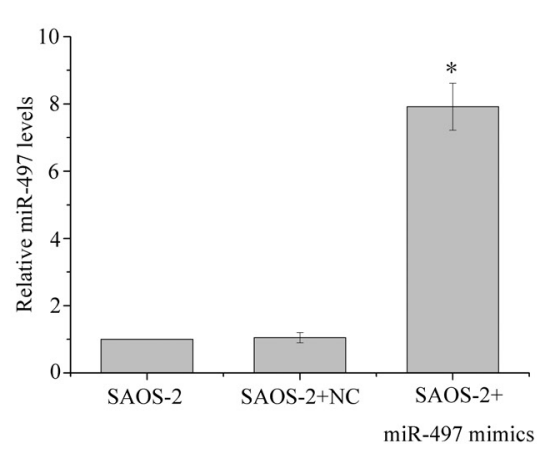

$\mathrm{C}$

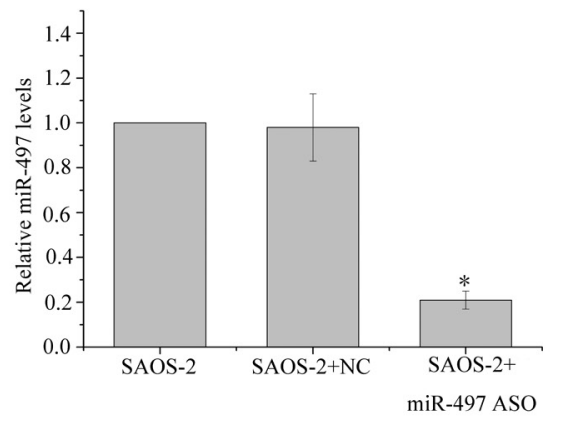

E

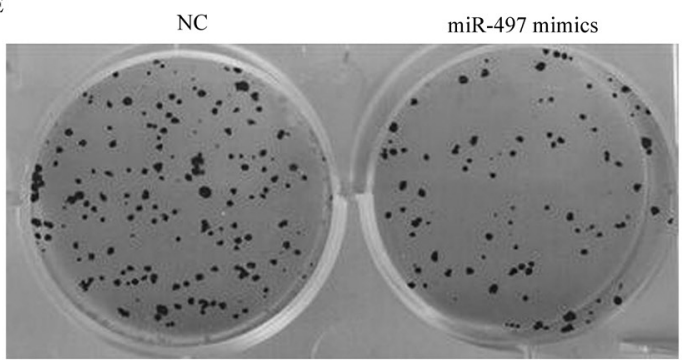

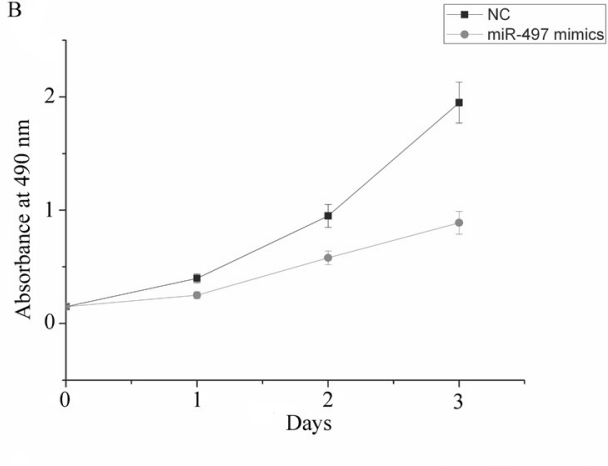

D

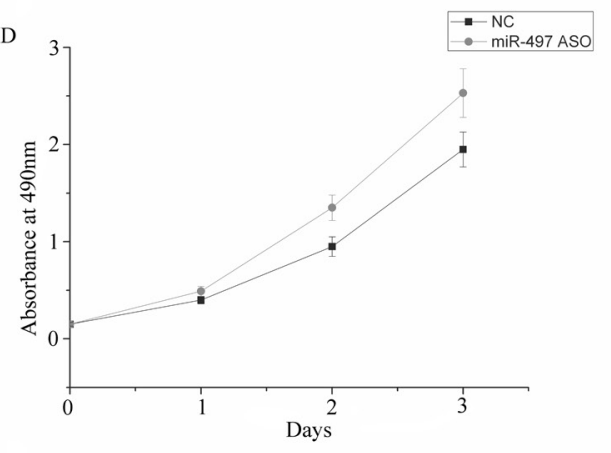

F

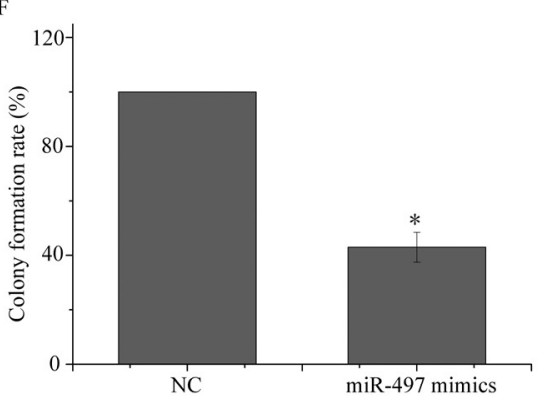

Fig. 2. Overexpression of miR-497 inhibits osteosarcoma cell proliferation. A: Expression of miR-497 was determined in Saos- 2 cells after miR-497 mimics transfection compared to controls. B: The growth curve of Saos- 2 cells after miR-497 mimics transfection compared to controls. C: Expression of miR-497 was determined in Saos- 2 cells after miR-497 ASO transfection compared to controls. D: The growth curve of Saos-2 cells after miR-497 ASO transfection compared to controls. E, F: The colony formation ability was determined in Saos- 2 cells tansfected with miR-497 mimics or NC. * Compared to NC group, $\mathrm{P}<0.05$.

VEGFA is a direct target of miR-497

We examined miR-497 potential targets by searching the TargetScan database. Among the candidates targets, 3'-UTR of human VEGFA contains a putative region (nucleotides 292299) that matches to the seed sequence of miR-497(Fig.4A). Next, to investigate whether the repression of VEGFA by miR-497 is mediated by direct interaction of miR-497 with VEGFA3'-UTR, we constructed luciferase reporters with wild-type (pGL3-VEGFA-wild) and mutated 3'-UTR of VEGFA (pGL3-VEGFA- mut). As shown in Fig. 4B, luciferase activity of the pGL3VEGFA-wild reporter was significantly suppressed in miR-497 mimic group compared with negative control group. Compared with the controls, miR-497 significantly decreased the VEGFA mRNA expression level (Fig.4C). And the VEGFA protein level was also significantly decreased by transfection of miR-497 (Fig. 4D, 4E). The results confirmed that VEGFA is a direct target of miR-497. 
A

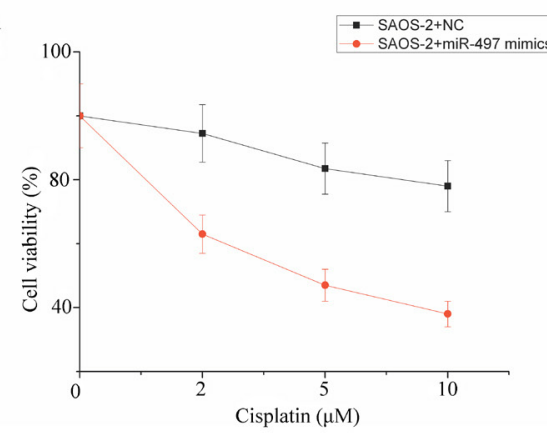

C
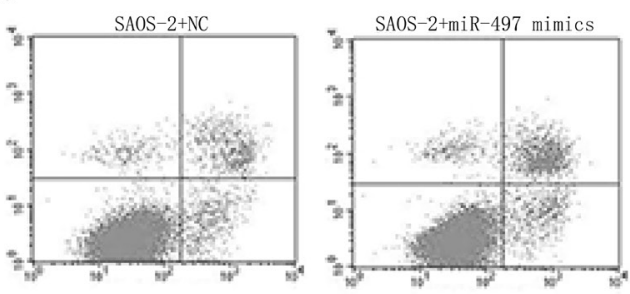

E
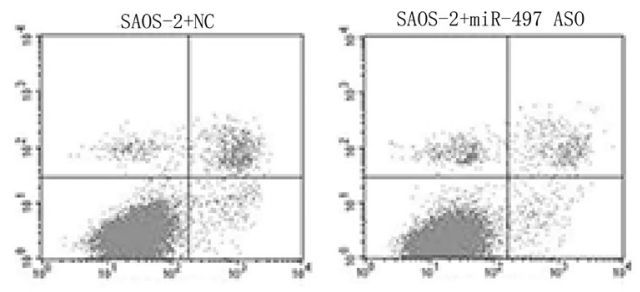

G

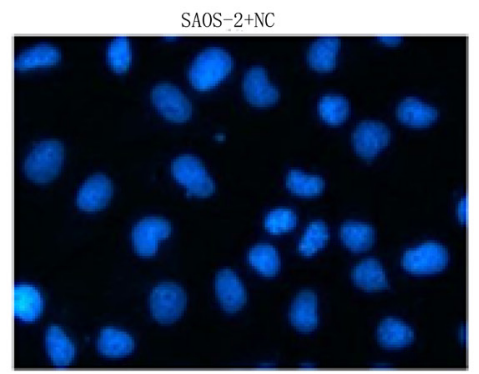

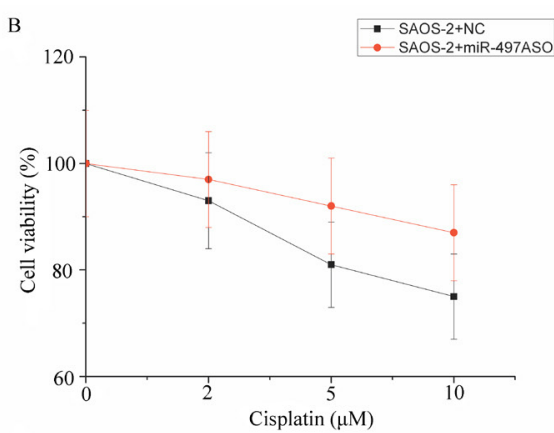

D
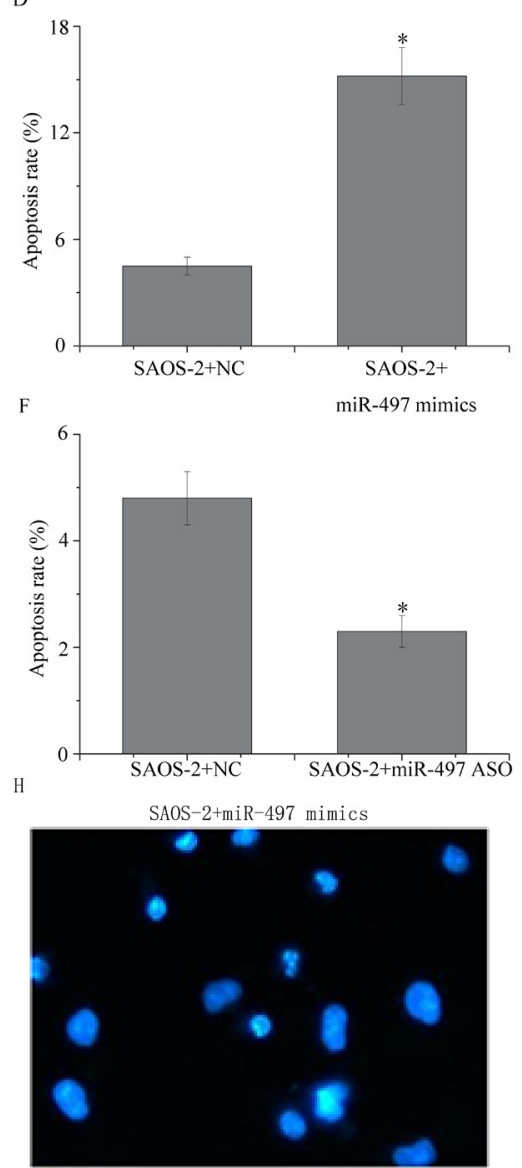

Fig. 3. miR-497 was involved in cisplatin resistance in SAOS-2 cells. (A) The Saos- 2 cells transfected with miR-497 mimics were treated with cisplatin for $48 \mathrm{~h}$. Percent survival was determined using the MTT assay. (B) The Saos-2 cells transfected with miR-497 ASO were treated with cisplatin for $48 \mathrm{~h}$. Percent survival was determined using the MTT assay. (C, D) Annexin-V/PI apoptosis assay of control, miR-497 mimics transfected SAOS-2 cells treated with $2 \mu \mathrm{M}$ of cisplatin for $48 \mathrm{~h}$. (E, F) Annexin-V/PI apoptosis assay of control, miR-497 ASO transfected SAOS-2 cells treated with $2 \mu \mathrm{M}$ of cisplatin for $48 \mathrm{~h}$. G, H: The apoptosis was determined by Hoechst 33258 stain assay in Saos-2 cells tansfected with miR-497 mimics or NC. * Compared to $\mathrm{NC}$ group, $\mathrm{P}<0.05$.

MiR-497 inhibits tumor growth and sensitizes SAOS-2 cells to cisplatin via VEGFA/PI3K/ AKT pathway

Several studies revealed that inactivation of VEGF /PI3K/Akt pathway could augment BCL-2 and CCND1 expression. The CCK-8 assay demonstrated that LY294002 (PI3K inhibitor, 


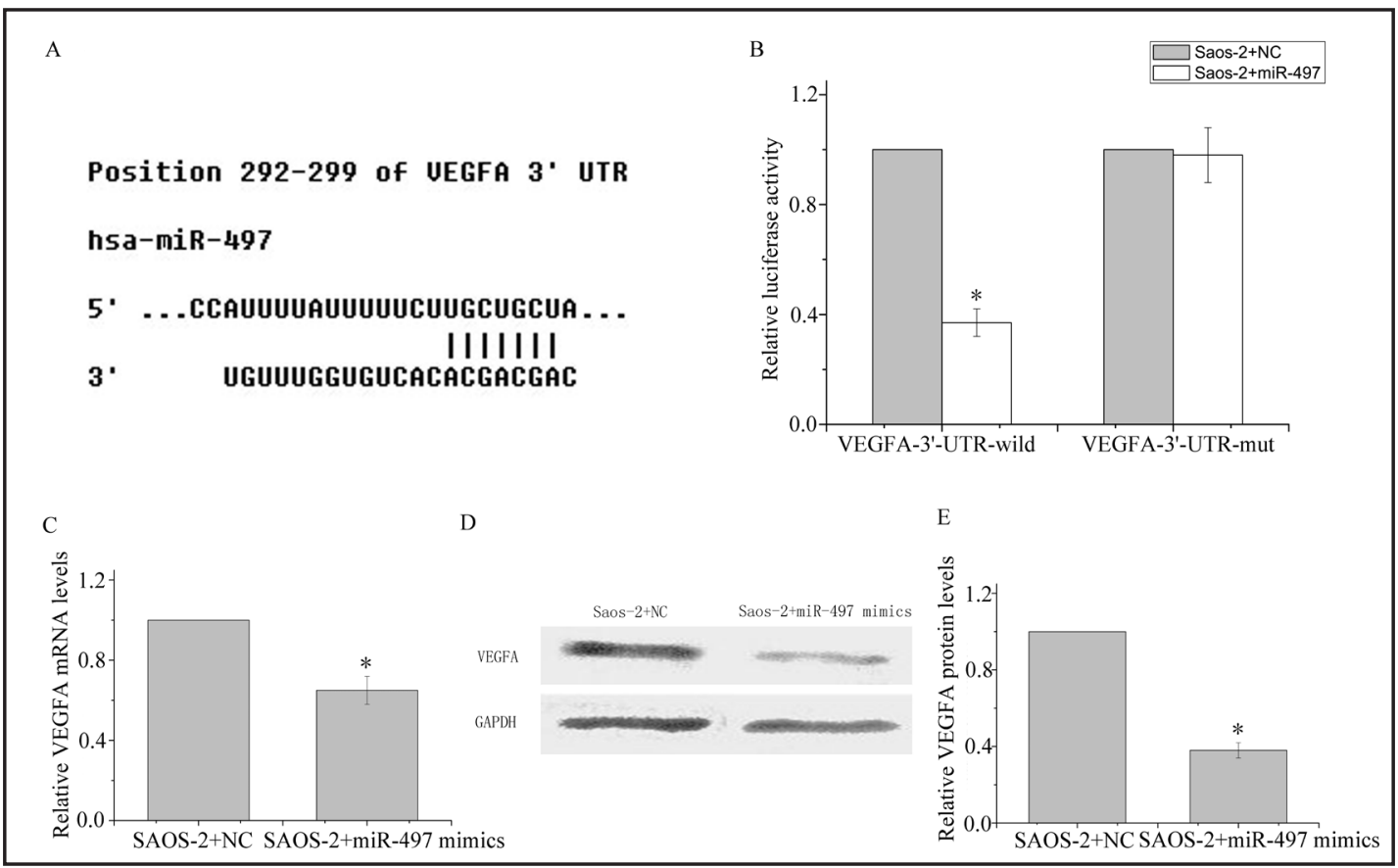

Fig. 4. A: Sequence alignments between miR-497 and 3'UTR of mRNAs. B: Luciferase activity of the wild-type VEGFA-3'-UTR reporter was significantly suppressed in miR-497 mimic group compared with negative control group C: miR-497 significantly decreased the VEGFA mRNA expression level. D, E: the VEGFA protein level was also significantly decreased by transfection of miR-497. * Compared to NC group, $\mathrm{P}<0.05$.

block the PI3K/AKT pathway) abolished miR-497 inhibitors-inducing cisplatin resistance in Saos-2 cells (Fig. 5A). To confirm whether miR-497 inhibits tumor growth and modulates sensitivity to cisplatin through VEGF /PI3K/Akt pathway in SAOS-2 cells, we detected proteins expression of t-AKT, p-AKT, BCL-2 and CCND1. After 48 hours, protein levels of BCL2 and CCND1 were increased by miR-497 inhibitors transfection in SAOS- 2 cells. To further establish the axis of miRNA-497/VEGF /PI3K/AKT, cells were transfected with miR-497 inhibitors and treated with LY294002 at $30 \mu \mathrm{M}$. LY294002 treatment inhibited phospho-AKT protein levels induced by miR-497 inhibitors, and abolished miR-497 inhibitors-inducing AKT activation leading to subsequent decrease of BCL-2 and CCND1 protein levels (Fig. 5B). These results suggested that downregulation of miR-497 could augment BCL-2 and CCND1 expression through AKT activation in SAOS-2 cells.

Re-expression of miR-497 could modulate the sensitivity to cisplatin in SAOS-2 cells in vivo

To investigate whether re-expression of miR-497 is useful in modulating the sensitivity to cisplatin in vivo or not, we established SAOS-2 cells subcutaneous xenograft tumor model in nude mice. Twelve days after inoculation, the mice were treated with cisplatin alone or in combination with miR-497 mimics. In the combination group, as detected by real-time quantitative reverse transcription, miR-497 levels were markedly increased in tumor when compared with negative control group (Fig. 6A), suggesting that miR-497 mimics effectively increased miR-497 expression in tumor in vivo. The growth curves of xenografts showed that tumor masses in mice who received combination therapy grew more slowly than those in cisplatin alone group. At the start of treatment (10 days after inoculation), there was no statistically significant difference between these two groups. Five days after the first administration of cisplatin, there was a significant difference in the median tumor size between these two groups $(\mathrm{P}<0.05)$ and it increased as the treatment continued. 35 days after inoculation, the tumor size in mice who received combination 


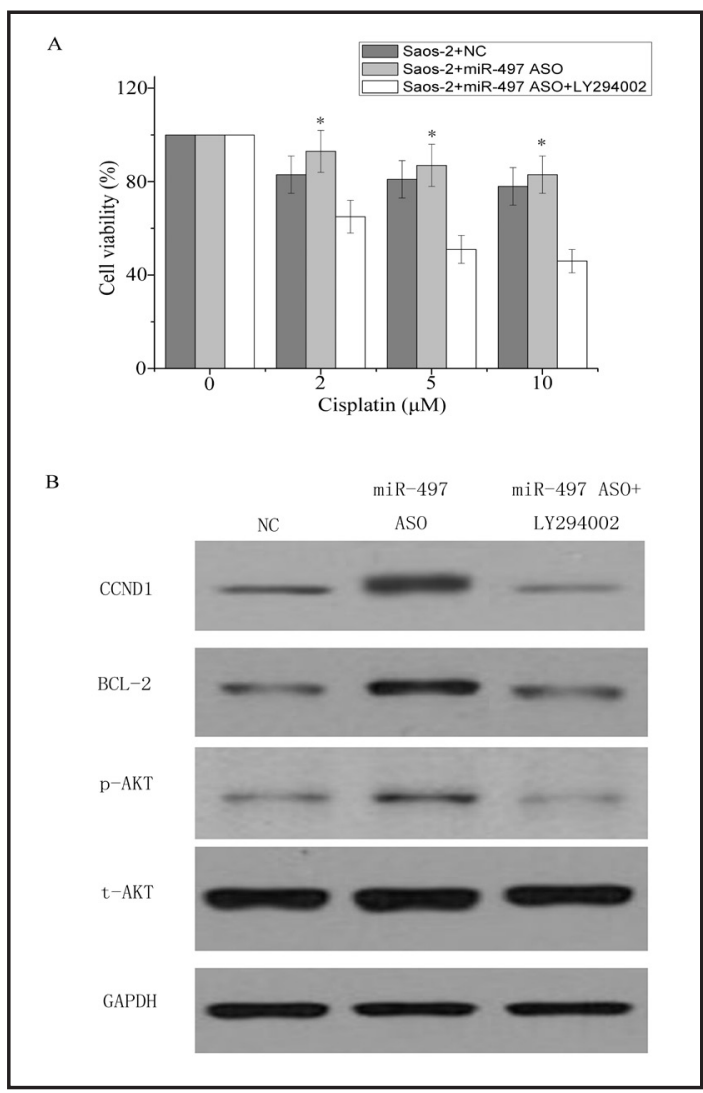

Fig. 5. MiR-497 modulated the activation of PI3K/ AKT pathway. A: LY294002 abolished miR-497 ASO-inducing cisplatin resistance in Saos-2 cells. B: LY294002 abolished miR-497 ASO-inducing AKT activation leading to subsequent decrease of BCL-2 and CCND1 protein levels.

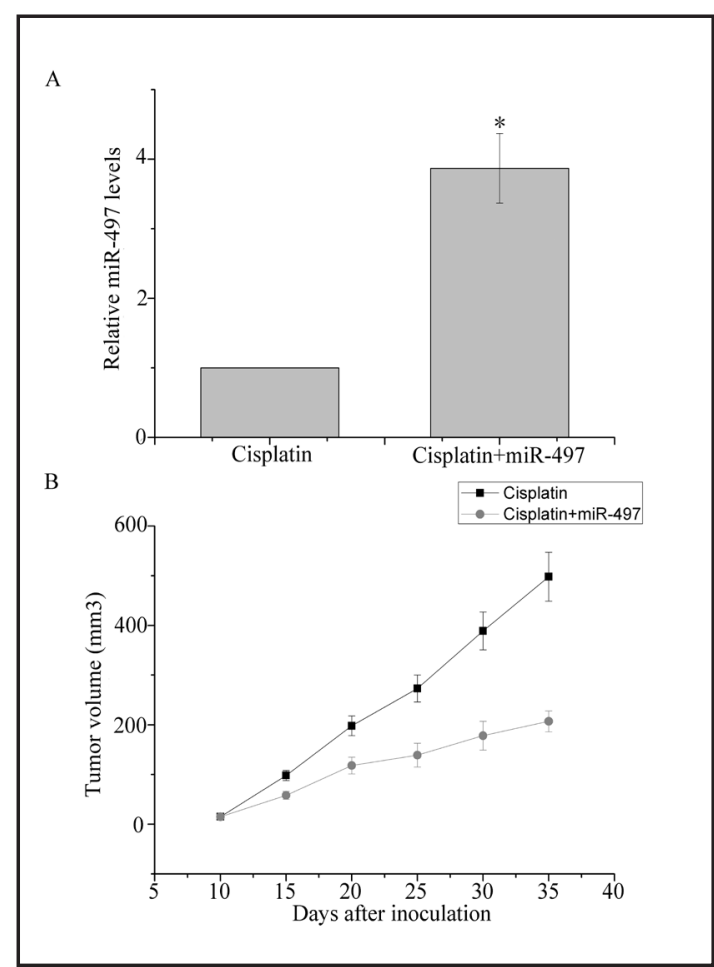

Fig. 6. miR-497 modulated the sensitivity to cisplatin in SAOS-2 cells in vivo.A: miR-497 levels were markedly increased in tumor when compared with control group. B: the tumor size in mice who received combination therapy was smaller than tumor size in those given cisplatin alone. ${ }^{*} P<0.05$.

therapy $\left(198.7 \pm 100.9 \mathrm{~mm}^{3}\right)$ was smaller than tumor size in those given cisplatin alone $\left(498.3 \pm 283.7 \mathrm{~mm}^{3}\right)$. There is a significant difference between these two groups $(\mathrm{P}<0.05)$.

\section{Discussion}

Accumulating evidence has demonstrated that miRNAs may exert functions as oncogenes or tumor suppressors in human cancers via modulating the expression of their targets $[18,19]$. Therefore, better understanding of the physiological and disease-associated mechanisms of these small, single-stranded RNAs may provide a new way for diagnosis and therapy for various diseases, including cancers. In this study, we provide important evidence that miR-497 plays an important role in tumor proliferation and sensitivity to cisplatin in osteosarcoma.

Some studies have been conducted to investigate the important roles of microRNAs in OS using miRNA expression profiles. A study from Maire et al. performed miRNA expression profiling of seven OS samples and suggest that miRNAs provide a novel regulatory mechanism for the expression of specific genes and pathways relevant to osteosarcoma [20]. MiR-497 belongs to the miR-15/16/195/424/497 family. Members of this miR family share the same 3' untranslated region (3'UTR) binding seed sequence and 
have been were found to sensitize cisplatin-resistant cells to apoptosis. Previous studies showed that miR-497 functions as a tumor-suppressor and is down-regulated in several kinds of tumors, including cervical cancer, colorectal cancer, and prostate cancer [11, 21, 22]. Similar results were also observed in breast cancer, in which miR-497 was significantly downregulated in breast cancer and miR-497 suppressed breast cancer cells proliferation and invasion $[23,24]$. A recent study by Shen et al. suggested that miR-497 inhibited cellular growth, enhanced the percentage of early apoptotic cells and inducedG0/G1 cell phase arrest of MCF-7 cancer cells and the up-regulation of miR-497 resulted in the decrease of Bcl-w at the mRNA and protein levels [25]. However, whether miR-497 regulates target genes to control osteosarcoma cells growth and sensitivity to cisplatin is still unknown. In the present study, we found miR-497 was significantly down-regulated in osteosarcoma tissue samples and the osteosarcoma cell line SAOS-2 compared with adjacent normal tissues and normal human osteoblasts. Furthermore, overexpression of miR-497 significantly inhibited SAOS-2 cells proliferation and enhanced the sensitivity to cisplatin. These results demonstrated that miR-497 plays a role as a tumor-suppressor in osteosarcoma.

Constitutive activation of PI3K/Akt pathway has been found in a variety of diseases, including many malignancies and contributes to tumor cell survival $[26,27]$. Inhibition of PI3K-Akt pathway by pharmacologic approach could lead to restoration of drug sensitivity in many cancer cell lines including osteosarcoma cells [28-30].The up-regulation of this signal pathway was also suggested to increase resistance to chemotherapy agents [27]. But the role of PI3K-Akt pathway in cisplatin sensitivity in different cancer cell lines is inconsistent. PI3K/AKT signal pathway has been shown to be crucial for the survival, proliferation, invasion, and migration of human cancer cells induced by VEGFA [31]. Zhu et al. found that increased expression of miR-126 enhanced the sensitivity of NSCLC cells to chemotherapy agents through negative regulation of the VEGF/PI3K/Akt/MRP1 signaling pathway [32]. Results from another study suggest that Eag1 siRNA could inhibit tumor growth and angiogenesis in osteosarcoma through modulating the activation of VEGF/ PI3K/AKT signaling [33]. Similar to their results, we found the protein levels of VEGFA were up-regulated in osteosarcoma tissues and in the osteosarcoma cell line SAOS-2 compared with adjacent nontumorous osteosarcoma tissues and normal human osteoblasts. The results demonstrated that the overexpression of VEGFA was associated with osteosarcoma. Bioinformatics analysis confirmed that VEGFA was a direct target gene of miR-497 in this study. LY294002 treatment inhibited phospho-Akt protein levels induced by miR-497 inhibitors, and abolished miR-497 inhibitors-inducing Akt activation leading to subsequent decrease of BCL-2 and CCND1 protein levels. These results suggested that miR-497 could modulate proliferation and apoptosis through VEGFA/PI3KAKT pathway in SAOS-2 cells.

In summary, our study demonstrated that downregulation of miR-497 induced cell survival and cisplatin resistance at least through PI3K/Akt pathway in human osteosarcoma by direct targeting VEGFA. We also provided direct evidence that the miR-497 mimic was a new potential therapeutic approach in osteosarcoma. Further studies are needed to confirm the roles of miR-497 and other miRNAs in osteosarcoma cells.

\section{Disclosure Statement}

The authors declare no conflict of interest.

\section{References}

1 Ottaviani G, Jaffe N: The epidemiology of osteosarcoma. Cancer Treat Res 2009;152:3-13.

2 Desandes E: Survival from adolescent cancer. Cancer Treat Rev 2007;33:609-615.

3 Chou AJ, Gorlick R: Chemotherapy resistance in osteosarcoma: current challenges and future directions. Expert Rev Anticancer Ther 2006;6:1075-1085. 


\section{Cellular Physiology Cell Physiol Biochem 2015;36:2051-2062 \begin{tabular}{ll|l} 
and Biochemistry Publisned onlIne: July 17,2015 & $\begin{array}{l}\text { C) } 2015 \text { S. Karger AG, Basel } \\
\text { www.karger.com/cpb }\end{array}$ \\
\hline
\end{tabular}}

4 Bartel DP: MicroRNAs: genomics, biogenesis, mechanism, and function. Cell 2004;116:281-297.

5 Pogribny IP, Filkowski JN, Tryndyak VP, Golubov A, Shpyleva SI, Kovalchuk O: Alterations of microRNAs and their targets are associated with acquired resistance of MCF-7 breast cancer cells to cisplatin. Int J Cancer 2010;127:1785-1794.

6 Yu ZW, Zhong LP, Ji T, Zhang P, Chen WT, Zhang CP: MicroRNAs contribute to the chemoresistance of cisplatin in tongue squamous cell carcinoma lines. Oral Oncol 2010;46:317-322.

7 Port M, Glaesener S, Ruf C, Riecke A, Bokemeyer C, Meineke V, Honecker F, Abend M: Micro-RNA expression in cisplatin resistant germ cell tumor cell lines. Mol Cancer 2011;10:52.

$>8$ Imanaka Y, Tsuchiya S, Sato F, Shimada Y, Shimizu K, Tsujimoto G: MicroRNA-141 confers resistance to cisplatin-induced apoptosis by targeting YAP1 in human esophageal squamous cell carcinoma. J Hum Genet 2011;56:270-276.

-9 Bian HB, Pan X, Yang JS, Wang ZX, De W: Upregulation of microRNA-451 increases cisplatin sensitivity of non-small cell lung cancer cell line (A549). J Exp Clin Cancer Res 2011;30:20.

10 Hummel R, Watson DI, Smith C, Kist J, Michael MZ, Haier J, Hussey DJ: Mir-148a improves response to chemotherapy in sensitive and resistant oesophageal adenocarcinoma and squamous cell carcinoma cells. J Gastrointest Surg 2011;15:429-438.

11 Guo ST, Jiang CC, Wang GP, Li YP, Wang CY, Guo XY, Yang RH, Feng Y, Wang FH, Tseng HY, Thorne RF, Jin L, Zhang XD: MicroRNA-497 targets insulin-like growth factor 1 receptor and has a tumour suppressive role in human colorectal cancer. Oncogene 2013;32:1910-1920.

-12 Flavin RJ, Smyth PC, Laios A, O'Toole SA, Barrett C, Finn SP, Russell S, Ring M, Denning KM, Li J, Aherne ST, Sammarae DA, Aziz NA, Alhadi A, Sheppard BL, Lao K, Sheils OM, O'Leary JJ: Potentially important microRNA cluster on chromosome 17p13.1 in primary peritoneal carcinoma. Mod Pathol 2009;22:197205.

-13 Finnerty JR, Wang WX, Hebert SS, Wilfred BR, Mao G, Nelson PT: The miR-15/107 group of microRNA genes: evolutionary biology, cellular functions, and roles in human diseases. J Mol Biol 2010;402:491-509.

14 Zhu W, Zhu D, Lu S, Wang T, Wang J, Jiang B, Shu Y, Liu P: miR-497 modulates multidrug resistance of human cancer cell lines by targeting BCL2. Med Oncol 2012;29:384-391.

15 Marie PJ, Lomri A, Sabbagh A, Basle M: Culture and behavior of osteoblastic cells isolated from normal trabecular bone surfaces. In Vitro Cell Dev Biol 1989;25:373-380.

16 Figueras A, Arbos MA, Quiles MT, Vinals F, Germa JR, Capella G: The impact of KRAS mutations on VEGF-A production and tumour vascular network. BMC Cancer 2013;13:125.

17 Zhang J, Han C, Wu T: MicroRNA-26a promotes cholangiocarcinoma growth by activating beta-catenin. Gastroenterology 2012;143:246-256 e248.

18 Dang X, Ma A, Yang L, Hu H, Zhu B, Shang D, Chen T, Luo Y: MicroRNA-26a regulates tumorigenic properties of EZH2 in human lung carcinoma cells. Cancer Genet 2012;205:113-123.

19 Qi P, Cheng SQ, Wang H, Li N, Chen YF, Gao CF: Serum microRNAs as biomarkers for hepatocellular carcinoma in Chinese patients with chronic hepatitis B virus infection. PLoS One 2011;6:e28486.

20 Maire G, Martin JW, Yoshimoto M, Chilton-MacNeill S, Zielenska M, Squire JA: Analysis of miRNA-gene expression-genomic profiles reveals complex mechanisms of microRNA deregulation in osteosarcoma. Cancer Genet 2011;204:138-146.

21 Luo M, Shen D, Zhou X, Chen X, Wang W: MicroRNA-497 is a potential prognostic marker in human cervical cancer and functions as a tumor suppressor by targeting the insulin-like growth factor 1 receptor. Surgery 2013;153:836-847.

22 Wang L, Li B, Li L, Wang T: MicroRNA-497 suppresses proliferation and induces apoptosis in prostate cancer cells. Asian Pac J Cancer Prev 2013;14:3499-3502.

23 Li D, Zhao Y, Liu C, Chen X, Qi Y, Jiang Y, Zou C, Zhang X, Liu S, Wang X, Zhao D, Sun Q, Zeng Z, Dress A, Lin MC, Kung HF, Rui H, Liu LZ, Mao F, Jiang BH, Lai L: Analysis of MiR-195 and MiR-497 expression, regulation and role in breast cancer. Clin Cancer Res 2011;17:1722-1730.

24 Lehmann U, Streichert T, Otto B, Albat C, Hasemeier B, Christgen H, Schipper E, Hille U, Kreipe HH, Langer F: Identification of differentially expressed microRNAs in human male breast cancer. BMC Cancer 2010;10:109.

25 Shen L, Li J, Xu L, Ma J, Li H, Xiao X, Zhao J, Fang L: miR-497 induces apoptosis of breast cancer cells by targeting Bcl-w. Exp Ther Med 2012;3:475-480. 


\section{Cellular Physiology Cell Physiol Biochem 2015;36:2051-2062

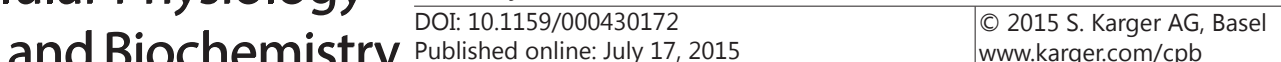 www.karger.com/cpb

-26 Zollinger A, Stuhmer T, Chatterjee M, Gattenlohner S, Haralambieva E, Muller-Hermelink HK, Andrulis M, Greiner A, Wesemeier C, Rath JC, Einsele H, Bargou RC: Combined functional and molecular analysis of tumor cell signaling defines 2 distinct myeloma subgroups: Akt-dependent and Akt-independent multiple myeloma. Blood 2008;112:3403-3411.

27 Peng DJ, Wang J, Zhou JY, Wu GS: Role of the Akt/mTOR survival pathway in cisplatin resistance in ovarian cancer cells. Biochem Biophys Res Commun 2010;394:600-605.

28 Chen KF, Chen HL, Tai WT, Feng WC, Hsu CH, Chen PJ, Cheng AL: Activation of phosphatidylinositol 3-kinase/Akt signaling pathway mediates acquired resistance to sorafenib in hepatocellular carcinoma cells. J Pharmacol Exp Ther 2011;337:155-161.

29 She QB, Solit D, Basso A, Moasser MM: Resistance to gefitinib in PTEN-null HER-overexpressing tumor cells can be overcome through restoration of PTEN function or pharmacologic modulation of constitutive phosphatidylinositol 3'-kinase/Akt pathway signaling. Clin Cancer Res 2003;9:4340-4346.

-30 Wang H, Luo QF, Peng AF, Long XH, Wang TF, Liu ZL, Zhang GM, Zhou RP, Gao S, Zhou Y, Chen WZ: Positive feedback regulation between Akt phosphorylation and fatty acid synthase expression in osteosarcoma. Int J Mol Med 2014;33:633-639.

-31 Abid MR, Guo S, Minami T, Spokes KC, Ueki K, Skurk C, Walsh K, Aird WC: Vascular endothelial growth factor activates PI3K/Akt/forkhead signaling in endothelial cells. Arterioscler Thromb Vasc Biol 2004;24:294-300.

-32 Zhu X, Li H, Long L, Hui L, Chen H, Wang X, Shen H, Xu W: miR-126 enhances the sensitivity of non-small cell lung cancer cells to anticancer agents by targeting vascular endothelial growth factor A. Acta Biochim Biophys Sin (Shanghai) 2012;44:519-526.

-33 Wu J, Wu X, Zhong D, Zhai W, Ding Z, Zhou Y: Short Hairpin RNA (shRNA) Ether a go-go 1 (Eag1) inhibition of human osteosarcoma angiogenesis via VEGF/PI3K/AKT signaling. Int J Mol Sci 2012;13:12573-12583. 